\title{
Jejunal Infusion of Levodopa-Carbidopa Intestinal Gel Versus Oral Administration of Levodopa-Carbidopa Tablets in Japanese Subjects with Advanced Parkinson's Disease: Pharmacokinetics and Pilot Efficacy and Safety
}

\author{
Ahmed A. Othman ${ }^{1,2} \cdot$ Krai Chatamra ${ }^{3}$ Mohamed-Eslam F. Mohamed ${ }^{1}$. \\ Sandeep Dutta ${ }^{1} \cdot$ Janet Benesh $^{3} \cdot$ Masayoshi Yanagawa $^{4} \cdot$ Masahiro Nagai $^{5}$
}

Published online: 15 April 2015

(c) The Author(s) 2015. This article is published with open access at Springerlink.com

\begin{abstract}
Background and Objective Oral levodopa-carbidopa (LC-oral) treatment in advanced Parkinson's disease (PD) is associated with motor complications due to large fluctuations in levodopa plasma concentrations. Levodopacarbidopa intestinal gel (LCIG) provides individualized continuous levodopa-carbidopa delivery through intrajejunal infusion. This study evaluated the pharmacokinetics, safety, and efficacy of LCIG relative to LC-oral in Japanese subjects with advanced PD.

Methods Subjects with advanced PD were converted from their anti-PD medications to individually optimized doses of LC-oral (10:1 levodopa:carbidopa ratio) for 28 days (baseline; period 1) followed by switching to intrajejunal infusion of LCIG (4:1 ratio) for 21 days (period 2). Pharmacokinetics, adverse events (AEs), and efficacy were assessed.

Results Eight patients were enrolled. Six received LCIG and four reported at least one $\mathrm{AE}$ [most common: fall $(33.3 \%)$, dyskinesia $(33.3 \%)$ ]; one discontinued due to an AE. The average daily dose was $1230 / 123$ and $1370 / 342 \mathrm{mg}$ levodopa/carbidopa for LC-oral and LCIG, respectively, at
\end{abstract}

Ahmed A. Othman

ahmed.othman@abbvie.com

1 Clinical Pharmacology and Pharmacometrics, AbbVie, 1 North Waukegan Road, Building AP13A-3, North Chicago, IL 60064, USA

2 Department of Pharmaceutics, Faculty of Pharmacy, Cairo University, Cairo, Egypt

3 Neuroscience Development, AbbVie, North Chicago, IL, USA

4 AbbVie GK, Tokyo, Japan

5 Clinical Therapeutic Trial Center, Ehime University Hospital, Ehime, Japan the end of each period. The degree of fluctuation and intrasubject variability of levodopa plasma concentrations were 5.5- and 4-fold lower, respectively, with LCIG than with LCoral. Levodopa bioavailability was $99 \%$ for LCIG relative to LC-oral. Compared with baseline, LCIG decreased "Off" time (2.68 h, $P=0.002)$ and increased "On" time without troublesome dyskinesia $(2.35 \mathrm{~h}, P=0.006)$ in the PD Diary $^{\odot}$. With the small sample size, no statistically significant changes were seen on other efficacy endpoints.

Conclusions In Japanese subjects with advanced PD, LCIG resulted in an improved pharmacokinetic profile that appeared to be associated with reduced motor complications compared with LC-oral. These results extend previous findings in mainly Caucasian populations.

\section{Key Points}

Continuous jejunal infusion of levodopa-carbidopa intestinal gel (LCIG) provides a viable option to patients with advanced Parkinson's disease (PD) who experience motor complications that are inadequately controlled by standard oral therapy.

Pharmacokinetic and efficacy benefits of continuous jejunal infusion of LCIG have been previously demonstrated in Western patients.

This study demonstrates that LCIG jejunal infusion results in lower fluctuations in levodopa concentrations and shows comparable bioavailability to oral administration in Japanese subjects with advanced PD. The improved pharmacokinetic profile with LCIG infusion appears to be associated with reduced motor complications. 


\section{Introduction}

Levodopa is the gold-standard treatment of Parkinson's disease (PD) and is eventually used by all PD patients [1]. However, the majority of PD patients who receive levodopa for 5-10 years develop disabling motor complications (motor fluctuations and dyskinesias) [2]. Development of motor complications is attributed to the narrowing of the therapeutic window between brain dopamine concentrations that precipitate abnormal involuntary movements (dyskinesias) and those that ameliorate the parkinsonian symptoms as the disease progresses [3, 4]. Maintaining levodopa plasma concentrations, and consequently brain dopamine concentrations, within a patient-specific narrow therapeutic window is challenging. Intermittent frequent administration of standard oral levodopa formulations leads to fluctuating plasma concentrations due erratic gastric emptying and the short half-life of levodopa [5-9].

Levodopa-carbidopa intestinal gel (LCIG; Duodopa ${ }^{\circledR}$; Duopa $^{\circledR}$; AbbVie, North Chicago, IL, USA) is a suspension of levodopa and carbidopa (20 and $5 \mathrm{mg} / \mathrm{mL}$, respectively) in an aqueous sodium carboxymethylcellulose gel. LCIG is infused through a portable pump (CADD-Legacy ${ }^{\circledR}$, Smith Medical, Minneapolis, MN, USA) directly into the jejunum via a nasojejunal (NJ) (short-term) or percutaneous gastrojejunostomy tube (long-term therapy) [10]. In the jejunum, levodopa is rapidly absorbed by an active carrier mechanism localized in the proximal small intestine [11]. By providing consistent levodopa plasma concentrations with patient-specific dosing, LCIG provides individualized continuous, rather than intermittent, stimulation of the dopaminergic receptors in the brain [3, 12]. LCIG has been shown to reduce the motor fluctuations, increase the 'On' time and decreased the incidence of dyskinesia in patients with advanced PD who have previously received oral treatment with levodopa for many years [13-16].

LCIG is approved for clinical use in more than 40 countries and is currently being studied in Japanese subjects. This was the first dedicated study to evaluate LCIG (4:1 levodopa:carbidopa ratio) relative to oral levodopa-carbidopa tablets (LC-oral, 10:1 levodopa:carbidopa ratio as approved in Japan) in Japanese subjects with advanced PD. The study was conducted in preparation for the Japan phase III clinical trial. Previous clinical trials of LCIG evaluated subjects of mainly (95-100 \%) Caucasian ethnicity and compared LCIG with oral 4:1 levodopa:carbidopa tablets $[3,12,14]$.

\section{Methods}

This study was an open-label, single-arm, baseline-controlled, multicenter study in subjects with advanced PD with severe motor complications despite optimized oral treatment with anti-PD medications. The study was designed to assess the pharmacokinetics, safety, and efficacy of LCIG relative to baseline treatment with LC-oral. Adult subjects (aged $\geq 30$ years) were eligible to participate in the study if they met the following criteria: a diagnosis of idiopathic PD according to the United Kingdom Parkinson's Disease Society (UKPDS) Brain Bank criteria, with their disease stage corresponding to 4 or 5 in the "Off" state (according to the modified Hoehn and Yahr classification) and which could not be satisfactorily controlled with optimized medical therapy; responsive to treatment with oral levodopa; severe motor fluctuations and dyskinesia; and a minimum daily "Off" time of $3 \mathrm{~h}$ during a continuous 16-h interval as supported by the subject PD diaries for 3 consecutive days including the portion of the day during which the subject was awake for the majority of the time (e.g., 6 a.m. to 10 p.m.). Additional inclusion criteria included being able to discontinue previous anti-PD medications other than the study drugs throughout the study and to keep a PD Diary ${ }^{\odot}$ [17] of "Off" time and dyskinesia.

Exclusion criteria included unclear diagnosis of PD, suspected diagnosis of other parkinsonian syndromes; other neurodegenerative diseases; history of neurosurgery for the treatment of PD or any other brain surgery; any neurological deficit that could possibly interfere with the study assessments; known hypersensitivity to levodopa, carbidopa, or any other constituent of LCIG or LC-oral tablets, or radiopaque material; concomitant narrow-angle glaucoma; a history of pheochromocytoma, Cushing's syndrome or malignant melanoma; having contraindications for the placement of an NJ tube; or any medical, surgical, and/or laboratory issues deemed by the investigator to be clinically significant.

The study [18] was conducted in five sites in Japan (National Center of Neurology and Psychiatry, Tokyo; Ehime University Hospital, Ehime; Wakayama Medical University Hospital, Wakayama; Juntendo University Hospital, Tokyo; and National Hospital Organization Utano Hospital, Kyoto). The study protocol and informed consent were approved by the institutional review board at each participating site.

\subsection{Study Procedures}

The study consisted of a screening period (maximum 14 days), a run-in period ( 28 days), an LCIG treatment period (21 days), and a follow-up period (7 days), as shown in Fig. 1. Subjects were hospitalized during the last 2 days of the run-in period (Days -2 and -1 ) and during the entire LCIG treatment period. The remainder of the study period was conducted under outpatient conditions.

During the run-in period, subjects were switched from their prior anti-PD medications to monotherapy with the 

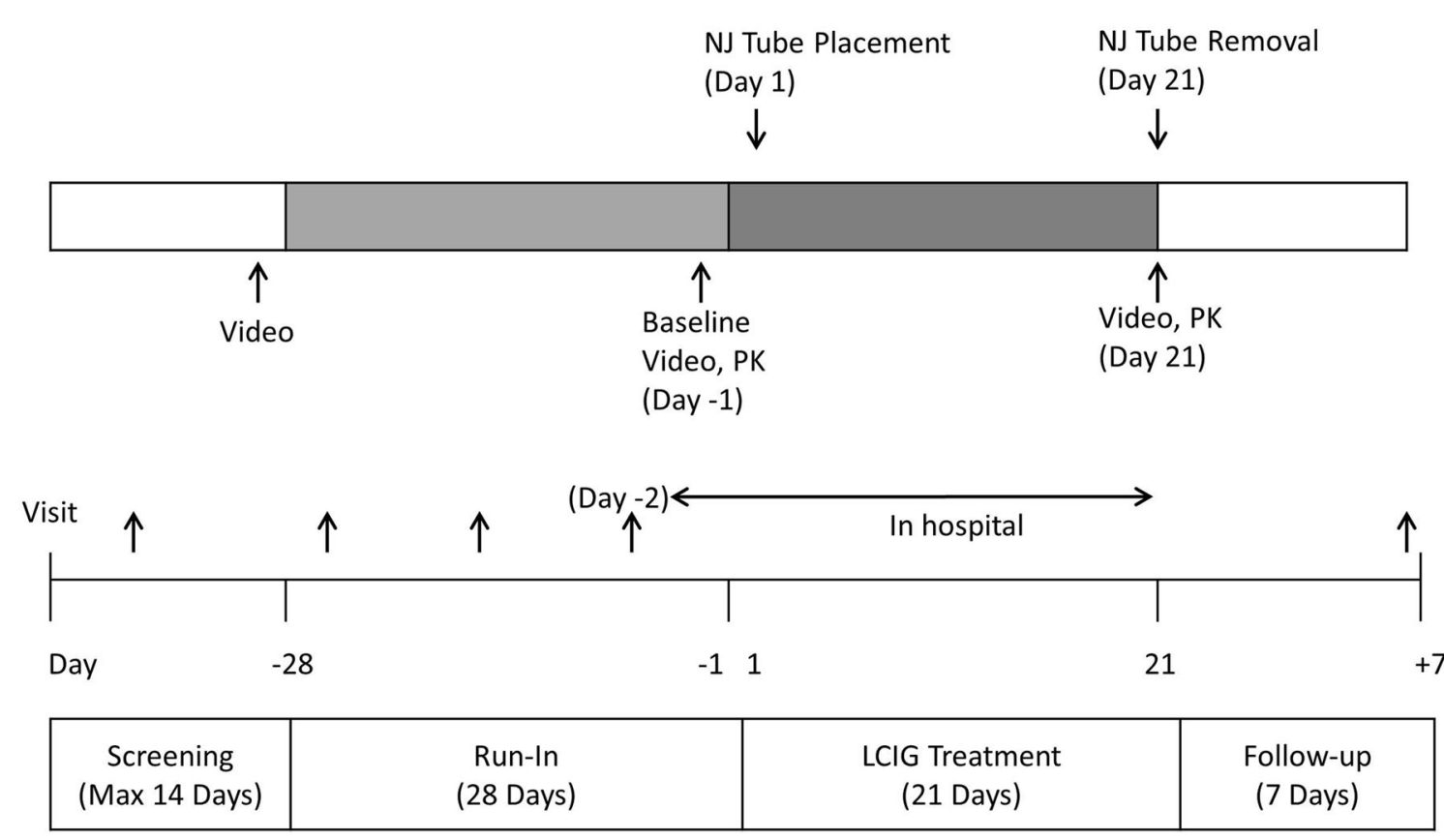

Fig. 1 Study design. $L C I G$ levodopa-carbidopa intestinal gel, Max maximum, $N J$ nasojejunal, $P K$ pharmacokinetics

LC-oral tablets (levodopa $100 \mathrm{mg}$ and carbidopa $10 \mathrm{mg}$; Menesit ${ }^{\circledR}$, Merck Sharp \& Dohme, Tokyo, Japan) and optimized on a six times daily schedule (every third hour; e.g., 6 a.m., 9 a.m., 12 p.m., 3 p.m., 6 p.m., 9 p.m.) during waking hours. The method for switching prior anti-PD medications to monotherapy with the LC-oral tablet was determined by the investigator based on the package insert of anti-PD medications and the LC-oral tablets. The run-in period was to allow the stabilization of PD symptoms following the elimination of previous long-acting anti-PD medications. Subjects used the PD and dosing diaries for 3 consecutive days prior to being hospitalized on Day -2 .

Subjects were trained on the appropriate use of the PD Diary ${ }^{\odot}$ during the screening period and the concordance rate between the subject's and investigator's assessment had to be at least $75 \%$ for the subject to enter the run-in period. All medications taken by the subject during the study [from signing the informed consent form until follow-up (Day +7)] were recorded.

On the baseline assessment day (Day -1; last day in the run-in period), subjects were recorded on video equipment for 1-2 min while performing a series of assigned movements (rest, finger taps, rapid alternating movement of hands, arising from chair and gait; including confirmation of postural stability) every $60 \mathrm{~min}$ from 8 a.m. to 5 p.m. Blood samples for pharmacokinetic analysis were collected. Subjects fasted overnight from the day before the baseline assessment day (Day -2). Water was allowed ad libitum. Subjects were requested not to consume excessive amounts of caffeine or caffeine-containing products per day and alcoholic beverages from $48 \mathrm{~h}$ prior to and throughout the confinement period. During the daytime, normal meals were allowed. Subjects were instructed to refrain from making any significant dietary changes throughout the duration of the study as a highprotein diet may reduce the effectiveness of levodopa administration independent of the mode of delivery.

An $\mathrm{NJ}$ tube was inserted in the morning of the first day of the LCIG treatment period (Day 1). Treatment with LCIG via the infusion pump was initiated after radiologically confirming the NJ tube tip location in the proximal jejunum. On Day 20, tube placement was checked, and if there was any indication that the tube was displaced, it was repositioned to the correct position.

LCIG was administered with an infusion pump via the $\mathrm{NJ}$ tube for $16 \mathrm{~h}$ a day (waking hours) directly into the proximal jejunum. Delivery of LCIG was via an aqueous suspension of levodopa $(20 \mathrm{mg} / \mathrm{mL})$ and carbidopa monohydrate $(5 \mathrm{mg} / \mathrm{mL})$ in $100 \mathrm{~mL}$ cassettes. LCIG was administered as a morning bolus $(5-10 \mathrm{~mL})$ followed by continuous infusion at a constant rate for the remainder of each patient's waking day (16 h), and, if needed, intermittent extra doses (patient initiated based on symptoms experience). The infusion was stopped overnight. The dose of LCIG on the first day of the LCIG treatment period (Day 1) was based on the daily dose of the oral levodopa component from the LC-oral tablets taken at baseline (Day -1 ). Dosing was optimized during the first 3-7 days of LCIG treatment to maximize the functional "On" time during the day by minimizing the number of "Off" 
(bradykinesia) episodes, the "Off" time, and the "On" time with troublesome dyskinesia. LCIG dose readjustments and patient-initiated extra doses were allowed except for on the last day of the LCIG treatment period (Day 21).

On Day 21, subjects were recorded on video equipment in the same manner as at baseline and blood samples for pharmacokinetic analysis were collected. Subjects fasted overnight (e.g., starting from 10 p.m. on the previous day) and dietary guidelines on Day 21 were similar to Day -1 . At the end of the LCIG treatment period, the NJ tube was removed after completion of all assessments required and the subjects were discharged from the hospital. Subjects were permitted, if necessary, to self-administer their typical night-time dosage of LC-oral tablets during the run-in and LCIG periods. However, no night-time intake of the LCoral tablet was allowed at least $3 \mathrm{~h}$ before the morning dosing of LC-oral or LCIG on the morning of the video recording day.

\subsection{Blood Sampling and Pharmacokinetic Analysis}

Blood samples (4 mL each) for pharmacokinetic analysis were collected at baseline (Day -1 ; last day of the run-in period) and end of LCIG treatment (Day 21). On Day -1 , blood samples were collected at time 0 and at 15, 30, 45, and $60 \mathrm{~min}$ following the morning dose and every $30 \mathrm{~min}$ thereafter for $12 \mathrm{~h}$. On Day 21, blood samples were collected at the same timepoints as for Day -1 and every $2 \mathrm{~h}$ from hours 12 to 16 relative to the start of the infusion.

Plasma concentrations of levodopa, carbidopa, and 3-Omethyldopa were determined by liquid chromatography with tandem mass spectrometry (LC-MS/MS) as previously described [3]. The analytical assay was validated over the concentration ranges of 10-5000, 0.5-250, and 25-25,000 ng/mL for levodopa, carbidopa, and 3-Omethyldopa, respectively. In-study between-run variability [\% coefficient of variation $(\% \mathrm{CV})]$ was $\leq 9.8 \%$ and the mean absolute bias was $\leq 5.2 \%$ for all three analytes.

Pharmacokinetic parameters for levodopa, carbidopa, and 3-O-methyldopa were estimated using non-compartmental methods. These included the maximum observed plasma concentration $\left(C_{\max }\right)$, time to $C_{\max }\left(t_{\max }\right)$, the minimum observed plasma concentration $\left(C_{\min }\right)$, area under the plasma concentration-time curve (AUC), and the average plasma concentrations $\left(C_{\mathrm{avg}}\right)$. Pharmacokinetic parameters were calculated for $12-\mathrm{h}$ intervals relative to administration of the first morning LC-oral dose on Day -1 and for 12- and 16-h intervals relative to the start of the LCIG infusion on Day 21. Additionally, $C_{\max }, C_{\min }$, and $C_{\text {avg }}$ values were calculated for the 2 - to 12 -h and 2- to 16-h intervals as applicable.

The degree of fluctuation for the 2- to 12-h interval relative to administration of first morning LC-oral dose and for the 2- to 12 - and 2- to 16 -h intervals relative to the start of the LCIG infusion were determined as $\left(C_{\max }-C_{\min }\right) / C_{\text {avg }}$ using the parameters calculated for the corresponding intervals. The 3-O-methyldopa to levodopa AUC from time zero to $12 \mathrm{~h}\left(\mathrm{AUC}_{12}\right)$ ratios were calculated. The inter- and intra-subject coefficient of variation for plasma concentrations were estimated for the 2- to 12-h intervals on Days -1 and 21 and for the 2- to 16-h interval on Day 21 using a linear mixed-effects model for $\log$ concentrations, with fixed effects for time and random effects for subject and occasion within subject. Levodopa bioavailability from LCIG compared to LC-oral was estimated by non-linear mixed-effects modeling with NONMEM $^{\circledR}$ (version 7.3; Icon Development Solutions, Ellicott City, MD, USA) using a previously published model for levodopa with both methods of administration [19].

\subsection{Efficacy Assessment}

Efficacy was assessed using the Treatment Response Scale (TRS) [13] calculated based on evaluation of video recordings made every $60 \mathrm{~min}$ from 8 a.m. to 5 p.m. on Day -1 (end of run-in LC-oral treatment period) and Day 21 (end of LCIG treatment period) by blinded raters (trained neurologists). Subjects were recorded on video equipment a total of ten times for 1-2 min every $60 \mathrm{~min}$ while performing a standardized sequence of motor tasks. As a blinded method for video evaluation, a dummy NJ tube was attached to the subject during video recording at the end of the LC-oral treatment (Day -1) and video recordings for each subject were provided to the raters in a random order. The three neurologists individually evaluated the video recordings. TRS I was calculated based on evaluation of the finger taps, rapid alternating movement of hands, arising from chair, gait, body bradykinesia and hypokinesia, and dyskinesia. Evaluation grades for TRS I were -3 , "severe Off"; -2 , "moderate Off"; -1, "mild Off"; 0, "On without dyskinesia"; and +1, "On with mild dyskinesia". The percentage of ratings in the range of -1 to +1 ("mild Off" to "On with mild dyskinesia") on the TRS I at the end of the LCIG treatment period (Day 21) compared with that of administration of LC-oral tablets at baseline (Day -1) was determined. Interrater reliability for TRS I was assessed by calculating a Kraemer $\kappa$ coefficient. A comparison of the efficacy between the end of the LCIG treatment period and baseline was carried out using a paired $t$ test at a two-sided significance level of $5 \%$. Other efficacy parameters included the mean daily "Off" time (h), the mean daily "On time with troublesome dyskinesia" and the mean daily "On time without troublesome dyskinesia" on the PD Diary ${ }^{\circledR}$ during 3 consecutive days (i.e., Days 18-20) prior to the end of the 
LCIG treatment period (Day 21) and during 3 consecutive days (i.e., Days -5 to -3 ) prior to baseline (Day -1 ).

\subsection{Safety}

All adverse events (AEs) reported from the first day of the run-in period until 30 days following completion or discontinuation of study drug administration were collected. Safety assessments included physical examination, neurological examination, vital signs, single ECG, blood sampling for clinical laboratories, hematology, blood chemistry, urinalysis, and scatoscopy.

The safety analysis included all subjects who had at least one dose of LC-oral study medication administered during the run-in period. LCIG safety analysis included all subjects who had had at least one dose of the LCIG study medication after the baseline assessment.

\subsection{Determination of Sample Size}

The sample size was estimated based on a previous openlabel, crossover study, which suggested that five subjects were required to have $80 \%$ power to detect a mean change of $14 \%$ in the percentage of ratings in the interval -1 to +1 on the TRS I at a two-sided $\alpha$-level of 0.05 [12]. Since this study was the first study of LCIG conducted in Japan and required subjects to receive LCIG via an NJ tube for a relatively longer period, the target number of subjects to be included in this study was eight, assuming a higher dropout rate $(30 \%)$ than previously seen.

\section{Results}

\subsection{Subjects' Disposition}

A total of eight (six male and two female) Japanese subjects with advanced PD (mean age of 65.4 years, range 58-72 years; mean bodyweight of $53.9 \mathrm{~kg}$, range $42.0-66.0 \mathrm{~kg}$; mean body mass index of $20.8 \mathrm{~kg} / \mathrm{m}^{2}$, range $17.5-23.9 \mathrm{~kg} / \mathrm{m}^{2}$; and mean disease duration of 14.9 years, range $7.8-19.9$ years) were enrolled in the study.

Two subjects prematurely discontinued the study in the run-in period [one subject did not meet the inclusion criteria at the beginning of the LCIG period and the other subject discontinued due to AEs (non-serious, probably not related) and withdrawal of informed consent] and six subjects were treated with LCIG. Out of the six subjects, one subject prematurely discontinued the study due to AEs (somatic hallucination, delusion, and auditory hallucination) in the LCIG treatment period and five subjects completed the study. In one subject, NJ tube was found to be displaced on Day 20, which was one of the three PD Diary ${ }^{\circledR}$ evaluation days. The tube was repositioned and the subject was included in pharmacokinetic, safety, and efficacy analyses, but was excluded from the PD Diary ${ }^{\circledR}$ evaluation.

\subsection{Pharmacokinetic Results}

The pharmacokinetic parameters for levodopa, carbidopa, and levodopa's metabolite 3-O-methyldopa are summarized in Table 1. Inter- and intra-subject variability (\%CV)

Table 1 Pharmacokinetic parameters (mean \pm standard deviation) of levodopa, carbidopa, and 3-O-methyldopa after administration of oral levodopa-carbidopa (10:1 levodopa:carbidopa ratio) tablets and jejunal infusion of levodopa-carbidopa intestinal gel (4:1 levodopa:carbidopa ratio) in Japanese subjects with advanced Parkinson's disease

\begin{tabular}{|c|c|c|c|c|c|c|}
\hline \multirow[t]{2}{*}{ Pharmacokinetic parameters } & \multicolumn{3}{|c|}{ LC-oral tablets $(n=5)$} & \multicolumn{3}{|c|}{ LCIG infusion $(n=5)$} \\
\hline & Levodopa & Carbidopa & 3-O-methyldopa & Levodopa & Carbidopa & 3-O-methyldopa \\
\hline Total study drug daily dose $\mathrm{e}^{\mathrm{a}}(\mathrm{mg})$ & $1230 \pm 246$ & $123 \pm 25$ & & $1370 \pm 353$ & $342 \pm 88$ & \\
\hline$t_{\max }(\mathrm{h})$ & $3.0 \pm 3.5$ & $7.8 \pm 2.8$ & $11 \pm 0.76$ & $1.0 \pm 0.50$ & $4.5 \pm 4.2$ & $11 \pm 0.79$ \\
\hline$C_{\max }(\mu \mathrm{g} / \mathrm{mL})$ & $5.96 \pm 0.768$ & $0.128 \pm 0.025$ & $9.27 \pm 2.17$ & $4.38 \pm 1.15$ & $0.273 \pm 0.066$ & $11.7 \pm 1.25$ \\
\hline$C_{\text {avg }}(\mu \mathrm{g} / \mathrm{mL})$ & $2.37 \pm 0.257$ & $0.079 \pm 0.015$ & $7.36 \pm 1.93$ & $2.87 \pm 0.663$ & $0.172 \pm 0.044$ & $9.80 \pm 1.23$ \\
\hline $\mathrm{AUC}_{12}(\mu \mathrm{g} \cdot \mathrm{h} / \mathrm{mL})$ & $28.4 \pm 3.08$ & $0.943 \pm 0.177$ & $88.3 \pm 23.1$ & $34.4 \pm 7.95$ & $2.07 \pm 0.522$ & $118 \pm 14.7$ \\
\hline $\mathrm{AUC}_{16}(\mu \mathrm{g} \cdot \mathrm{h} / \mathrm{mL})$ & & & & $46.7 \pm 10.7$ & $2.80 \pm 0.666$ & $165 \pm 21.2$ \\
\hline$C_{\min }(2-12 \mathrm{~h})(\mu \mathrm{g} / \mathrm{mL})$ & $0.734 \pm 0.425$ & $0.050 \pm 0.017$ & $5.72 \pm 1.53$ & $2.38 \pm 0.770$ & $0.130 \pm 0.035$ & $8.14 \pm 0.936$ \\
\hline Degree of fluctuation $(2-12 \mathrm{~h})^{\mathrm{b}}$ & $2.1 \pm 0.59$ & $0.97 \pm 0.20$ & $0.48 \pm 0.10$ & $0.38 \pm 0.16$ & $0.78 \pm 0.25$ & $0.35 \pm 0.07$ \\
\hline $\mathrm{M} / \mathrm{P}\left(\mathrm{AUC}_{12}\right)$ & & & $3.11 \pm 0.71$ & & & $3.53 \pm 0.70$ \\
\hline
\end{tabular}

$A U C_{x}$ area under the plasma concentration-time curve from time zero to $x \mathrm{~h}, C_{a v g}$ average plasma concentration, $C_{\max }$ maximum observed plasma concentration, $C_{\min }$ minimum observed plasma concentration, $L C I G$ levodopa-carbidopa intestinal gel, $L C$-oral oral levodopa-carbidopa, $M / P$ ratio of metabolite (3- $O$-methyldopa) to parent (levodopa), $t_{\max }$ time to $C_{\max }$

${ }^{\text {a }}$ Total dose between hours 0 and 16 on the day of pharmacokinetic assessment (Day -1 for LC-oral tablets, Day 21 for LCIG)

${ }^{\mathrm{b}}$ Degree of fluctuation calculated as $\left(C_{\max }-C_{\min }\right) / C_{\mathrm{avg}}$ 
Table 2 Inter- and intra-subject variability (\% coefficient of variation) for levodopa, carbidopa, and 3-O-methyldopa plasma concentrations during hours 2-12 following administration of the first morning oral levodopa-carbidopa tablet or initiation of levodopacarbidopa intestinal gel infusion

\begin{tabular}{|c|c|c|c|c|c|}
\hline \multirow[t]{2}{*}{ Analyte } & \multirow[t]{2}{*}{$n$} & \multicolumn{2}{|c|}{ Inter-subject CV (\%) } & \multicolumn{2}{|c|}{ Intra-subject CV (\%) } \\
\hline & & $\begin{array}{l}\text { LC-oral } \\
\text { tablets }\end{array}$ & LCIG & $\begin{array}{l}\text { LC-oral } \\
\text { tablets }\end{array}$ & LCIG \\
\hline Levodopa & 5 & 19 & 24 & 38 & 10 \\
\hline Carbidopa & 5 & 20 & 24 & 29 & 20 \\
\hline $\begin{array}{l}\text { 3-O- } \\
\text { methyldopa }\end{array}$ & 5 & 26 & 13 & 7 & 7 \\
\hline
\end{tabular}

Estimates based on a linear mixed-effects model for log concentration with fixed effects for time and random effects for subject and for occasion within subject

$C V$ coefficient of variation, $L C I G$ levodopa-carbidopa intestinal gel, $L C$-oral oral levodopa-carbidopa

for levodopa, carbidopa, and 3-O-methyldopa plasma concentrations during Hours 2-12 following administration of the first morning LC-oral tablet or initiation of LCIG intrajejunal infusion are presented in Table 2. Levodopa and carbidopa plasma concentrations [mean \pm standard deviation (SD)] versus time profiles after administration of LC-oral tablets and intrajejunal infusion of LCIG in Japanese subjects with advanced PD are presented in Fig. 2.

The average daily dose on the pharmacokinetic assessment day was 1230/123 and 1370/342 mg levodopa/carbidopa for LC-oral and LCIG, respectively. Levodopa and carbidopa mean $C_{\text {avg }}$ values were 2.37 and $0.079 \mu \mathrm{g} / \mathrm{mL}$ for LC-oral and 2.87 and $0.172 \mu \mathrm{g} / \mathrm{mL}$ for $\mathrm{LCIG}$, respectively. Degree of fluctuation and intra-subject variability in levodopa plasma concentrations were 5.5- and 4-fold lower, respectively, with LCIG than with LC-oral administration. LCIG resulted in approximately 2.2-fold higher exposure of carbidopa than administration of LC-oral tablets, which is consistent with the difference in the carbidopa dose in the two products. Following infusion termination on the night prior to LCIG pharmacokinetic assessment, none of the subjects received night-time doses of LC-oral tablets.

Using a previously developed pharmacokinetic model of levodopa with LCIG and LC-oral administration, levodopa bioavailability from intrajejunal infusion of LCIG relative to that with administration of LC-oral tablets was estimated to be $99.2 \%$ (95\% CI 96.5-102).

The pharmacokinetic parameters of levodopa, carbidopa, and 3-O-methyldopa after intrajejunal infusion of LCIG in Japanese subjects (current study) compared with previous observations in Caucasian subjects [3] are presented in Table 3. Comparison of levodopa and carbidopa plasma concentrations (mean and SD) versus time profile after intrajejunal infusion of LCIG in Japanese (current study) and Caucasian subjects [3] with advanced PD are presented in Fig. 3. The average daily levodopa/carbidopa LCIG dose in the Caucasian study was $1580 / 395 \mathrm{mg}$ compared with 1370/ $342 \mathrm{mg}$ in the present study.

\subsection{Efficacy}

A summary of the efficacy assessments using the TRS I video recordings and the PD Diary ${ }^{\circledR}$ is presented in Table 4.

The mean increase (improvement) in the "normal" state on TRS I by video assessment from LC-oral baseline to the end of LCIG treatment was numerically favorable but did not reach statistical significance. The Kraemer $\kappa$ coefficient for concordance between the three raters on TRS I was 0.22 .

PD Diary $\left.{ }^{(}\right)$results show that the mean change in the "Off" time from baseline to the end of LCIG treatment was reduction of $2.68 \mathrm{~h}(95 \% \mathrm{CI}-3.51$ to $-1.84, P=0.002)$. The mean increase in the "On" time without dyskinesia plus time with non-troublesome dyskinesia from baseline to the end of LCIG treatment was $2.35 \mathrm{~h}(95 \% \mathrm{CI}$ 1.28-3.43, $P=0.006)$. Other evaluated efficacy measures did not reach statistical significance.

\subsection{Safety and Tolerability}

The most frequently reported AEs in the run-in period (LCoral) were constipation and insomnia (three subjects each, $37.5 \%$ ), and nausea (two subjects, $25.0 \%$ ), and those in the LCIG treatment period were fall and dyskinesia (two subjects each, $33.3 \%$ ). The AEs reported in the study are common conditions associated with PD or are known AEs associated with LC-oral. Three AEs (thirst, nausea, and decreased appetite) occurred in one subject and were all assessed by the investigator as probably related to both study drug and NJ tube insertion.

\section{Discussion}

This open-label, single-arm, baseline-controlled, multicenter study evaluated the pharmacokinetics, safety, and efficacy of continuous intrajejunal infusion of LCIG (Duodopa $^{\circledR}$ ) in Japanese subjects with advanced PD. The study suggests that Japanese subjects with advanced PD can be safely converted from LC-oral (10:1 levodopa:carbidopa ratio) to LCIG (4:1 levodopa:carbidopa ratio). On the pharmacokinetic assessment day, consistent plasma concentrations of levodopa were achieved shortly after starting the daily intrajejunal infusion of LCIG and these consistent concentrations were maintained through the 


\section{Oral Levodopa-Carbidopa Tablets}

a
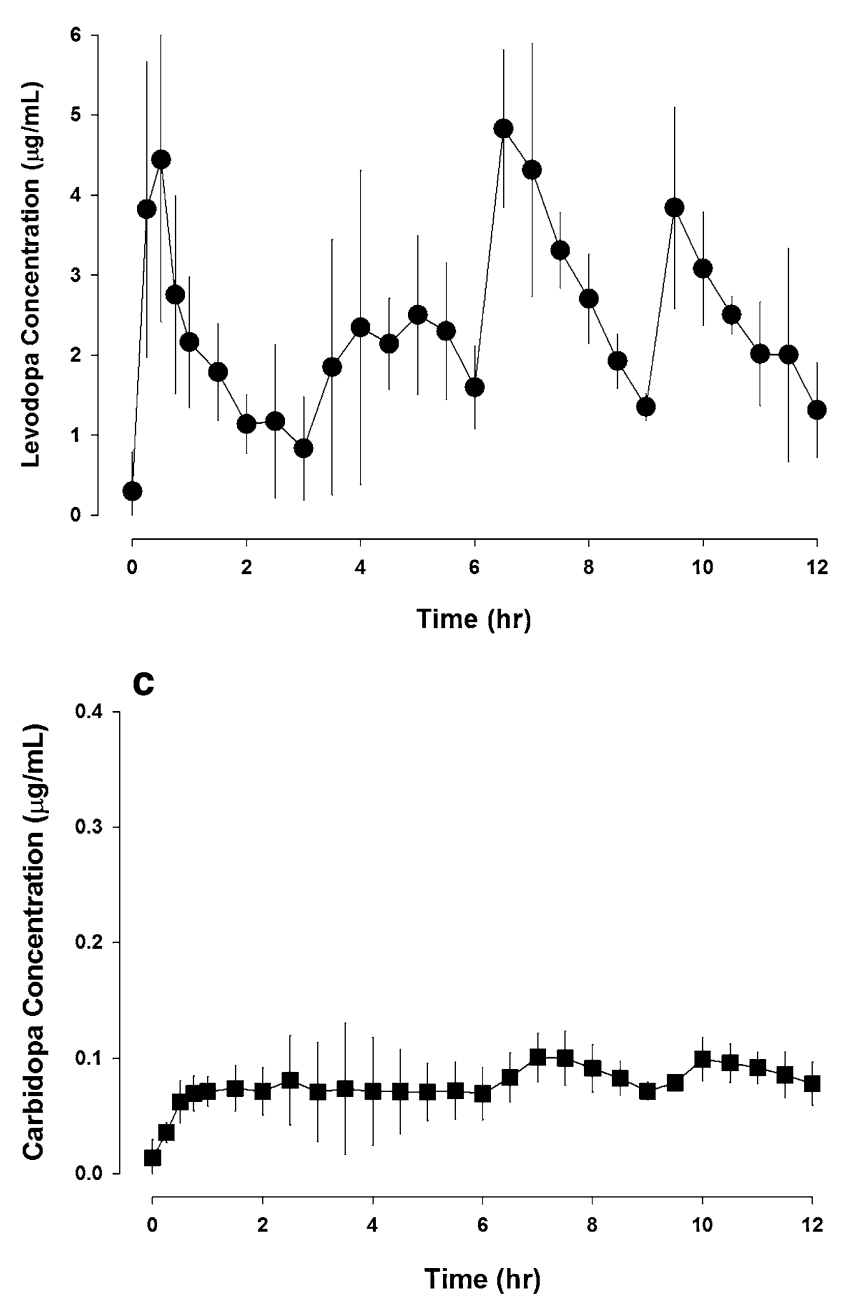

Intrajejunal LCIG Infusion

b
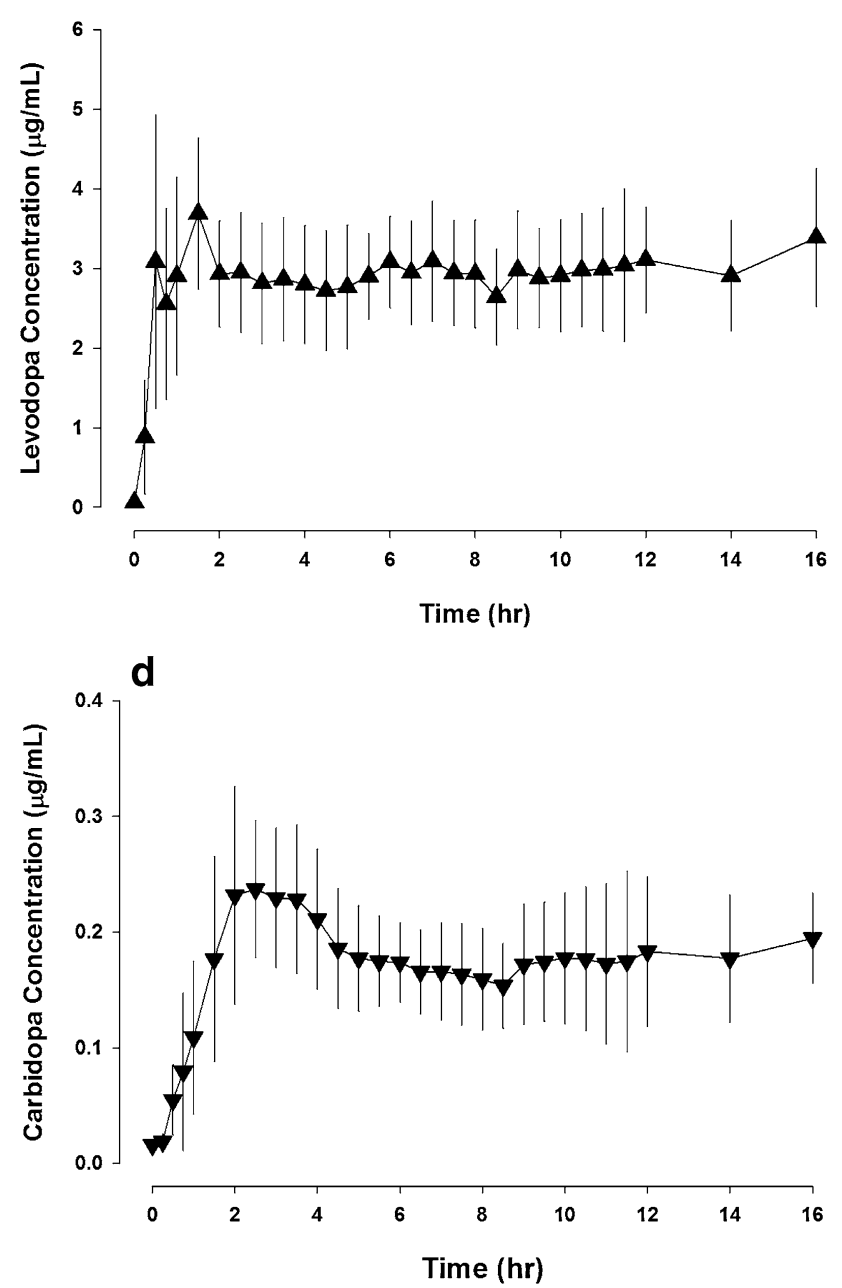

Fig. 2 Levodopa and carbidopa plasma concentrations (mean \pm standard deviation) versus time profiles after administration of oral levodopacarbidopa tablets (10:1 levodopa:carbidopa ratio; a, c) and intrajejunal infusion of levodopa-carbidopa intestinal gel (4:1 levodopa:carbidopa ratio; b, d) in Japanese subjects with advanced Parkinson's disease $(n=5)$. LCIG levodopa-carbidopa intestinal gel

daily infusion period (Fig. 2). Intra-subject variability in levodopa plasma concentrations was low (10\%) during LCIG treatment compared with LC-oral (38 \%; Table 2), and LCIG infusion displayed more than five-fold decrease in the degree of fluctuation in levodopa plasma concentrations compared with LC-oral (Table 1). The high fluctuations in levodopa concentrations with LC-oral treatment are attributed to levodopa's short half-life (approximately $1.5 \mathrm{~h}$ ) [20] coupled with its erratic gastric emptying and site-specific intestinal absorption. These limitations are addressed by continuous intrajejunal delivery of LCIG. This improved pharmacokinetic profile with LCIG intrajejunal infusion appeared to translate to better control of motor symptoms (Table 4).

Ethnic differences have been suggested in the response to levodopa-carbidopa therapy, and therefore in oral dosing requirements. Asian patients with $\mathrm{PD}$ appear to require 20-30\% lower doses of oral levodopa to control their symptoms and appear to develop dyskinesias more frequently than Caucasians $[21,22]$. This can be a result of higher levodopa bioavailability or greater pharmacodynamic sensitivity in Japanese subjects. We have previously characterized the pharmacokinetic profile of LCIG in Caucasian subjects with advanced PD [3]. The average daily levodopa/carbidopa LCIG dose in the Caucasian study was $1580 / 395 \mathrm{mg}$ compared to $1370 / 342 \mathrm{mg}$ in the present study. Intrajejunal infusion of individualized doses of LCIG in Japanese and Caucasian subjects resulted in comparably low fluctuations in levodopa concentrations and in superimposable mean levodopa plasma concentrations (Fig. 3; Table 3). The dose-normalized levodopa AUC during the 16-h infusion appeared to be $17 \%$ higher 
Table 3 Comparison of the pharmacokinetic parameters (mean \pm SD) of levodopa, carbidopa, and 3-O-methyldopa after intrajejunal infusion of levodopa-carbidopa intestinal gel in Japanese subjects (current study) and Caucasian subjects [3]

\begin{tabular}{|c|c|c|c|c|c|c|}
\hline \multirow[t]{2}{*}{ Pharmacokinetic parameters ${ }^{\mathrm{a}}$} & \multicolumn{3}{|c|}{ Japanese subjects $(n=5)$} & \multicolumn{3}{|c|}{ Caucasian subjects $(n=18)$} \\
\hline & Levodopa & Carbidopa & 3-O-methyldopa & Levodopa & Carbidopa & 3-O-methyldopa \\
\hline Total study drug daily dose ${ }^{\mathrm{b}}(\mathrm{mg})$ & $1370 \pm 353$ & $342 \pm 88$ & & $1580 \pm 403$ & $395 \pm 101$ & \\
\hline$C_{\max }(\mu \mathrm{g} / \mathrm{mL})$ & $4.38 \pm 1.15$ & $0.284 \pm 0.047$ & $12.9 \pm 2.26$ & $4.21 \pm 1.36$ & $0.371 \pm 0.149$ & $19.0 \pm 5.66$ \\
\hline$C_{\text {avg }}(\mu \mathrm{g} / \mathrm{mL})$ & $2.92 \pm 0.666$ & $0.175 \pm 0.042$ & $10.3 \pm 1.33$ & $2.91 \pm 0.836$ & $0.221 \pm 0.083$ & $17.1 \pm 4.99$ \\
\hline$C_{\min }(\mu \mathrm{g} / \mathrm{mL})$ & $0.061 \pm 0.027$ & $0.016 \pm 0.005$ & $7.78 \pm 0.632$ & $0.447 \pm 0.282$ & $0.103 \pm 0.067$ & $15.1 \pm 4.85$ \\
\hline $\mathrm{AUC}_{16}(\mu \mathrm{g} \cdot \mathrm{h} / \mathrm{mL})$ & $46.7 \pm 10.7$ & $2.80 \pm 0.666$ & $165 \pm 21.2$ & $46.5 \pm 13.3$ & $3.54 \pm 1.33$ & $273 \pm 79.8$ \\
\hline$C_{\min }(2-16 \mathrm{~h})(\mu \mathrm{g} / \mathrm{mL})$ & $2.38 \pm 0.77$ & $0.128 \pm 0.034$ & $8.14 \pm 0.936$ & $2.32 \pm 0.583$ & $0.167 \pm 0.073$ & $15.4 \pm 4.72$ \\
\hline Degree of fluctuation $^{\mathrm{c}}(2-16 \mathrm{~h})$ & $0.42 \pm 0.16$ & $0.87 \pm 0.19$ & $0.44 \pm 0.11$ & $0.52 \pm 0.20$ & $0.96 \pm 0.49$ & $0.21 \pm 0.11$ \\
\hline $\mathrm{M} / \mathrm{P}\left(\mathrm{AUC}_{16}\right)$ & & & $3.64 \pm 0.69$ & & & $5.97 \pm 1.09$ \\
\hline
\end{tabular}

$A U C_{16}$ area under the plasma concentration-time curve from time zero to $16 \mathrm{~h}, C_{a v g}$ average plasma concentration, $C_{\max }$ maximum observed plasma concentration, $C_{\text {min }}$ minimum observed plasma concentration, $M / P$ ratio of metabolite (3- $O$-methyldopa) to parent (levodopa)

${ }^{\text {a }}$ Parameters are for $0-16 \mathrm{~h}$ infusion interval unless otherwise specified

b Total dose between hours 0 and 16 on the day of pharmacokinetic assessment

${ }^{\mathrm{c}}$ Degree of fluctuation calculated as $\left(C_{\max }-C_{\min }\right) / \mathrm{C}_{\mathrm{avg}}$
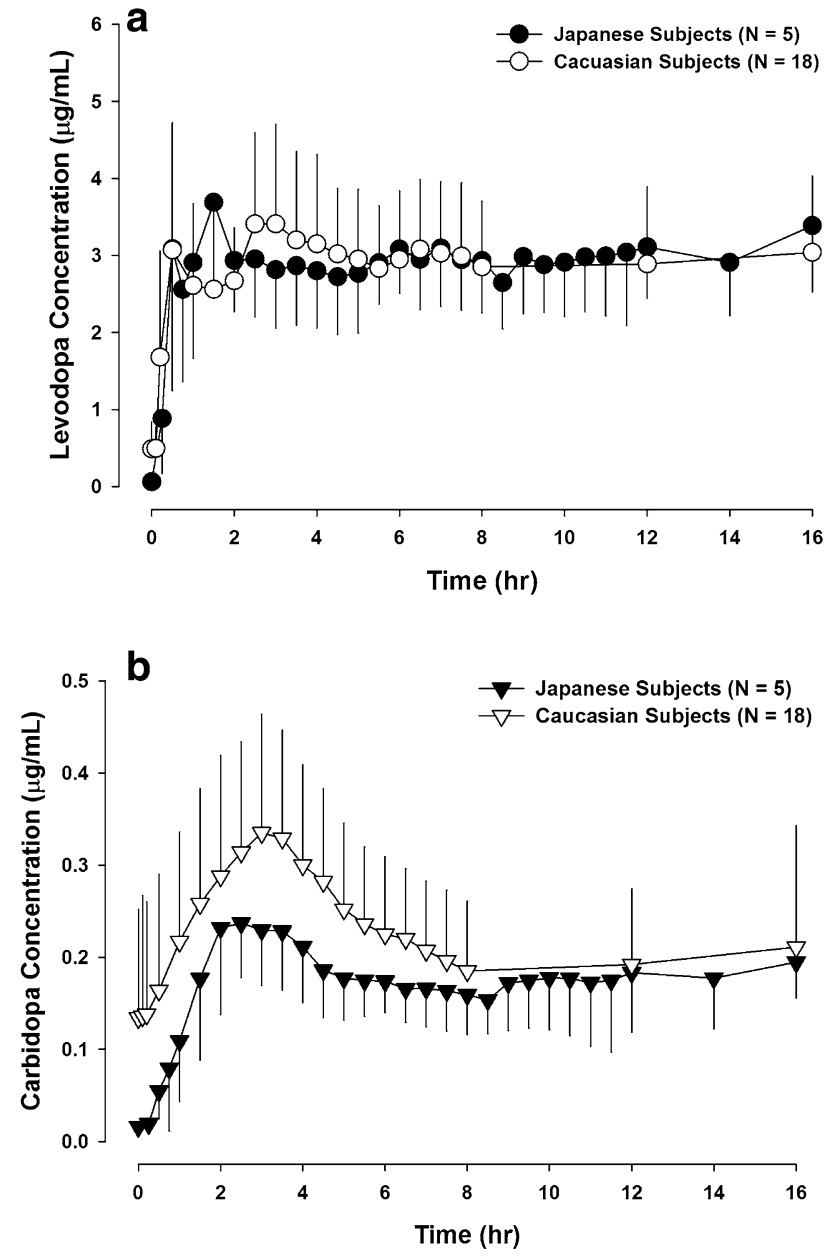

Fig. 3 Comparison of levodopa and carbidopa plasma concentrations (mean \pm standard deviation) versus time profiles after 16 -h intrajejunal infusion of LCIG in Japanese $(\mathbf{a} ; n=5)$ and Caucasian (b; $n=18$ ) subjects with advanced Parkinson's disease. Data for Caucasian subjects were previously reported [3] in Japanese subjects than previously observed in Caucasian subjects. Japanese subjects who participated in this study had lighter bodyweight, on average, compared with the Caucasian subjects evaluated in the previous study (54 vs. $66 \mathrm{~kg}$ average bodyweight). In the population pharmacokinetic analysis of levodopa data from Western LCIG Phase I and III studies (bodyweight range of 45-148 kg), we previously assessed bodyweight as a covariate for levodopa pharmacokinetic parameters and bodyweight was not found to be significantly correlated to levodopa clearance (which determines levodopa AUC; rather, bodyweight was better correlated with the levodopa volume of distribution) [19]. Findings from other levodopa population analyses were inconsistent regarding the contribution of bodyweight in explaining inter-subject variability in levodopa clearance, as previously discussed [19]. Overall, the small difference in levodopa dose-normalized AUC between the Japanese and Caucasian populations, if real, has no clinical relevance for LCIG since doses are individually titrated in each patient for optimal symptom control.

Carbidopa exposure in Japanese subjects appeared to be lower than in Caucasian subjects (Table 3), mainly because of lower carry-over carbidopa plasma concentrations (Fig. 3b). This is consistent with the lack of use of nighttime LC-oral tablets by Japanese patients in the current study, while most Caucasian patients in the previous study used night-time LC-oral tablets after discontinuation of the LCIG infusion on the night prior to the pharmacokinetic assessment day. Carbidopa reduces peripheral decarboxylation of levodopa to dopamine (which does not cross the blood-brain barrier) and directs more levodopa metabolism towards the catechol- $O$-methyl transferase (COMT) enzyme pathway (which generates the inactive metabolite, 
Table 4 Efficacy of levodopa-carbidopa intestinal gel (endpoint) relative to oral levodopa-carbidopa (baseline) using the "normal" state on Treatment Response Scale (TRS) I (video assessment) and the Parkinson's Disease Diary ${ }^{\odot}$ assessment

\begin{tabular}{|c|c|c|c|c|c|c|}
\hline Efficacy variable & $n$ & $\begin{array}{l}\text { Baseline (end of } \\
\text { LC-oral run-in } \\
\text { period) } \\
{[\text { mean } \pm \mathrm{SD}]}\end{array}$ & $\begin{array}{l}\text { End of LCIG } \\
\text { treatment period } \\
{[\text { mean } \pm \text { SD] }}\end{array}$ & $\begin{array}{l}\text { Change from baseline } \\
\text { (difference between } \\
\text { treatments) }[\text { mean } \pm \mathrm{SD}]\end{array}$ & $\begin{array}{l}95 \% \text { CI for change } \\
\text { from baseline [lower } \\
\text { bound, upper bound] }\end{array}$ & $P$ value $^{\mathrm{a}}$ \\
\hline \multicolumn{7}{|l|}{ TRS I assessment } \\
\hline TRS I (\%) "normal" state ${ }^{\mathrm{b}}$ & 5 & $62.7 \pm 18.2$ & $78.0 \pm 8.69$ & $15.3 \pm 14.26$ & $-2.37,33.0$ & 0.074 \\
\hline \multicolumn{7}{|c|}{ Parkinson's Disease Diary ${ }^{\odot}$ assessment $^{\mathrm{c}}$} \\
\hline Daily "Off" time (h) & 4 & $7.51 \pm 2.75$ & $4.83 \pm 2.51$ & $-2.68 \pm 0.52$ & $-3.51,-1.84$ & 0.002 \\
\hline $\begin{array}{l}\text { Daily "On" time without } \\
\text { dyskinesia }+ \text { time with non- } \\
\text { troublesome dyskinesia }(\mathrm{h})\end{array}$ & 4 & $7.74 \pm 1.48$ & $10.1 \pm 1.57$ & $2.35 \pm 0.68$ & $1.28,3.43$ & 0.006 \\
\hline $\begin{array}{l}\text { Daily "On" time with } \\
\text { troublesome dyskinesia (h) }\end{array}$ & 4 & $0.75 \pm 1.5$ & $1.08 \pm 1.6$ & $0.33 \pm 0.5$ & $-0.43,1.08$ & 0.261 \\
\hline
\end{tabular}

3-O-methyldopa). Exposure of 3-O-methyldopa was lower in Japanese subjects than in Caucasians (Table 3), which may be partially explained by the lower carbidopa plasma concentrations in Japanese subjects. Given the similarity across populations in plasma concentrations of the active moiety (levodopa) that mediates efficacy, the observed differences in carbidopa or 3-O-methyldopa exposure between populations are not clinically relevant.

Rivera-Calimlim and Reilly [22] suggested higher activity of the COMT enzyme in Japanese subjects. The 3-Omethyldopa exposure levels with LCIG use (same levodopa-carbidopa dose ratio across populations) are not supportive of higher COMT activity in Japanese subjects.

In the present study, the bioavailability of levodopa from LCIG relative to LC-oral tablets is estimated to be $99.2 \%$. This is consistent with levodopa's bioavailability estimate (97\%) for LCIG relative to LC-oral from a previous population pharmacokinetic analysis in mainly Caucasian subjects [19].

The potential benefit of the improved pharmacokinetic profile of levodopa with LCIG infusion was evaluated. A trend of improvement was observed in the percentage of subjects in the "normal" state on the TRS I video assessment, which did not reach statistical significance (Table 4). Lack of statistical significance is likely a result of the small sample size, the high inter-rater variability (Kraemer $\kappa$ coefficient for concordance between the three raters on TRS I was 0.22), and, potentially, the short evaluation duration ( $2 \mathrm{~min}$ ) within each of the assessment hours. Statistically significant improvements in "Off" time and "On" time without troublesome dyskinesia in the PD Diary ${ }^{\odot}$ evaluation were observed. The latter analysis excluded one subject for whom the NJ tube was dislocated (not in jejunum) on one of the PD Diary ${ }^{\odot}$ evaluation days.

The most frequently reported AEs in the LCIG treatment period were fall and dyskinesia, which are common conditions associated with Parkinson's disease or are known AEs associated with LC-oral. The majority of the AEs were assessed as mild or moderate in severity and were observed to decline over time. The safety assessment in this study is limited by the small number of subjects assessed during LCIG treatment (six subjects). While this number is sufficient to evaluate common AEs $(\geq 30 \%)$, it may have not been sufficient to identify less common ones. Additionally, LCIG was infused in this study through an NJ tube due to the exploratory nature of the study and the relatively short duration. However, in clinical practice, LCIG is infused through a percutaneous gastrojejunostomy tube inserted by a surgical procedure. In a recent relatively large randomized controlled, double-blind, double-dummy study of LCIG compared with LC-oral in a Western population, the majority of reported AEs during LCIG treatment were related to the surgical procedure or the intestinal tube [14].

Overall, this study suggests that Japanese subjects with advanced PD can be safely converted from LC-oral (10:1 ratio) to LCIG (4:1 ratio). LCIG intrajejunal infusion results in lower fluctuations in levodopa concentrations and comparable bioavailability as that of oral administration in Japanese subjects with advanced PD. The improved pharmacokinetic profile with LCIG infusion appeared to be associated with reduced motor complications. These results extend previous findings in mainly Caucasian populations. Based on the results from this study, a phase III study of 
LCIG in Japanese subjects was initiated and is currently ongoing (NCT01960842) [23].

Acknowledgments The authors thank the following investigators for participating in the study: Dr. M. Murata (National Center of Neurology and Psychiatry, Tokyo, Japan), Dr. T. Oeda (National Hospital Organization, Utano Hospital, Kyoto, Japan), Dr. Y. Shimo (Department of Neurology, Department of Research and Therapeutics for Movement Disorders, Juntendo University School of Medicine, Tokyo, Japan), and Dr. I. Nakanishi (Wakayama Medical University Kihoku Hospital, Wakayama, Japan).

Disclosures This study was sponsored by AbbVie. AbbVie contributed to the study design, research, and interpretation of data, writing, reviewing, and approving the manuscript. Drs. Othman, Chatamra, Mohamed, Dutta, and Benesh and Mr. Yanagawa are employees and shareholders of AbbVie. Dr. Nagai of Ehime University Hospital, Ehime, Japan was an investigator for the study. LCIG (Duodopa ${ }^{\circledR}$; Duopa ${ }^{\circledR}$; carbidopa-levodopa enteral suspension) is marketed by AbbVie in more than 40 countries and is currently being evaluated in clinical trials in Japan. The authors declare no other relationships or activities that could appear to have influenced the submitted work.

Open Access This article is distributed under the terms of the Creative Commons Attribution Noncommercial License which permits any noncommercial use, distribution, and reproduction in any medium, provided the original author(s) and the source are credited.

\section{References}

1. Hauser RA. Levodopa: past, present, and future. Eur Neurol. 2009;62(1):1-8.

2. Ahlskog JE, Muenter MD. Frequency of levodopa-related dyskinesias and motor fluctuations as estimated from the cumulative literature. Mov Disord. 2001;16(3):448-58.

3. Nyholm D, Odin P, Johansson A, Chatamra K, Locke C, Dutta S, et al. Pharmacokinetics of levodopa, carbidopa, and 3-Omethyldopa following 16-h jejunal infusion of levodopa-carbidopa intestinal gel in advanced Parkinson's disease patients. AAPS J. 2013;15(2):316-23.

4. Nyholm D. The rationale for continuous dopaminergic stimulation in advanced Parkinson's disease. Parkinsonism Relat Disord. 2007;13(Suppl):S13-7.

5. Nutt JG, Fellman JH. Pharmacokinetics of levodopa. Clin Neuropharmacol. 1984;7(1):35-49.

6. Hardoff R, Sula M, Tamir A, Soil A, Front A, Badarna S, et al. Gastric emptying time and gastric motility in patients with Parkinson's disease. Mov Disord. 2001;16(6):1041-7.

7. Kurlan R, Rothfield KP, Woodward WR, Nutt JG, Miller C, Lichter D, et al. Erratic gastric emptying of levodopa may cause "random" fluctuations of parkinsonian mobility. Neurology. 1988;38(3):419-21.

8. Nutt JG, Woodward WR, Hammerstad JP, Carter JH, Anderson JL. The "on-off" phenomenon in Parkinson's disease. Relation to levodopa absorption and transport. $\mathrm{N}$ Engl $\mathrm{J}$ Med. 1984;310(8):483-8.

9. Nutt JG, Woodward WR, Beckner RM, Stone CK, Berggren K, Carter $\mathrm{JH}$, et al. Effect of peripheral catechol-O- methyltransferase inhibition on the pharmacokinetics and pharmacodynamics of levodopa in parkinsonian patients. Neurology. 1994;44(5):913-9.

10. Nyholm D, Aquilonius SM. Levodopa infusion therapy in Parkinson disease: state of the art in 2004. Clin Neuropharmacol 2004;27(5):245-56.

11. Lennernas H, Nilsson D, Aquilonius SM, Ahrenstedt O, Knutson L, Paalzow LK. The effect of L-leucine on the absorption of levodopa, studied by regional jejunal perfusion in man. Br J Clin Pharmacol. 1993;35(3):243-50.

12. Nyholm D, Askmark H, Gomes-Trolin C, Knutson T, Lennernas $\mathrm{H}$, Nystrom C, et al. Optimizing levodopa pharmacokinetics: intestinal infusion versus oral sustained-release tablets. Clin Neuropharmacol. 2003;26(3):156-63.

13. Nyholm D, Nilsson Remahl AI, Dizdar N, Constantinescu R, Holmberg B, Jansson R, et al. Duodenal levodopa infusion monotherapy vs oral polypharmacy in advanced Parkinson disease. Neurology. 2005;64(2):216-23.

14. Olanow CW, Kieburtz K, Odin P, Espay AJ, Standaert DG, Fernandez $\mathrm{HH}$, et al. Continuous intrajejunal infusion of levodopa-carbidopa intestinal gel for patients with advanced Parkinson's disease: a randomised, controlled, double-blind, doubledummy study. Lancet Neurol. 2014;13(2):141-9.

15. Fernandez HH, Standaert DG, Hauser RA, Lang AE, Fung VSC, Klostermann F, et al. Levodopa-carbidopa intestinal gel in advanced Parkinson disease: final 12-month, open-label results. Mov Disord. 2014. doi:10.1002/mds.26123.

16. Slevin JT, Fernandez HH, Zadikoff C, Hall C, Eaton S, Dubow J, et al. Long-term safety and maintenance of efficacy of levodopacarbidopa intestinal gel: an open-label extension of the doubleblind pivotal study in advanced Parkinson's disease patients. J Parkinsons Dis. 2015;5(1):165-74.

17. Hauser RA, Friedlander J, Zesiewicz TA, Adler CH, Seeberger $\mathrm{LC}$, O'Brien CF, et al. A home diary to assess functional status in patients with Parkinson's disease with motor fluctuations and dyskinesia. Clin Neuropharmacol. 2000;23(2):75-81.

18. Abbott Japan Co., Ltd. Study of safety, tolerability, pharmacokinetics, and efficacy of ABT-SLV187 in subjects with advanced Parkinson's disease [ClinicalTrials.gov identifier NCT01479127]. US National Institutes of Health, ClinicalTrials.gov. http://www.clinicaltrials.gov. Accessed 18 Mar 2015.

19. Othman AA, Dutta S. Population pharmacokinetics of levodopa in subjects with advanced Parkinson's disease: levodopa-carbidopa intestinal gel infusion vs. oral tablets. Br J Clin Pharmacol. 2014;78(1):94-105.

20. Yeh KC, August TF, Bush DF, Lasseter KC, Musson DG, Schwartz S, et al. Pharmacokinetics and bioavailability of Sinemet CR: a summary of human studies. Neurology. 1989;39(11 Suppl 2):25-38.

21. Wood AJ, Zhou HH. Ethnic differences in drug disposition and responsiveness. Clin Pharmacokinet. 1991;20(5):350-73.

22. Rivera-Calimlim L, Reilly DK. Difference in erythrocyte catechol- $O$-methyltransferase activity between Orientals and Caucasians: difference in levodopa tolerance. Clin Pharmacol Ther. 1984;35(6):804-9.

23. AbbVie. A study to assess the efficacy, safety and tolerability of ABT-SLV187 monotherapy in subjects with advanced Parkinson's disease (PD) and persistent motor complications, despite optimized treatment with available anti-parkinsonian medications [ClinicalTrials.gov identifier NCT01960842]. US National Institutes of Health, ClinicalTrials.gov. http://www.clinicaltrials.gov. Accessed 18 Mar 2015. 